\title{
Personality Characteristics in Individual and Team Sports
}

\author{
$1^{\text {st }}$ Hermawan Pamot Raharjo \\ Physical Education Department \\ Universitas Negeri Semarang \\ Semarang, Indonesia \\ hermawan_pamot@yahoo.com
}

\author{
$2^{\text {nd }}$ Donny Wira Yudha Kusuma \\ Physical Education Department \\ Universitas Negeri Semarang \\ Semarang, Indonesia \\ donnywirayudhakusuma@mail.unnes.ac.id
}

\author{
$3^{\text {rd }}$ Mugiyo Hartono \\ Physical Education Department \\ Universitas Negeri Semarang \\ Semarang, Indonesia \\ mugiyohartono@mail.unnes.ac.id
}

\begin{abstract}
This study compares the personality characteristics of athletes in individual and team sports. 197 athletes (105 individual, 92 team, 131 males, and 66 females) completed the SPQ-20 Sport Personality Questionnaire. This questionnaire is composed of four sub- scales of 20 personality traits. The data were collected by Sport Personality Questionnaire 20 (SPQ-20) and the statistical analysis was performed with SPSS software. In order to describe the data, the description used to compare the average is the t-test. The results revealed that individual sport athletes scored significantly higher only on flow than team sport athletes. The team sport athletes scored significantly higher on conscientiousness, self-awareness, and ethics than what the individual sport athletes did. No significant difference was found between two groups on achievement, adaptability, competitiveness, visualization, intuition, goal setting, pressure management, self-efficacy, failure fear control, stress management, emotions, self-talk, empathy, relationships, aggressiveness, and impression management. It can be concluded that the personality characteristics of athletes are different between individual and team sports.
\end{abstract}

Keywords—personality characteristics, athletes, sports

\section{INTRODUCTION}

Personality is a determinant of achievement for sport, and is reliable for individuals who can help suit certain types of sports [1]. The research in the psychological aspects show that there are personality differences between individual and team sport has been study by many researchers. The differences between team and individual sport athletes are significantly higher on sociotropy and autonomy [2]. Another study revealed that individual sport athletes scored significantly higher on conscientiousness and autonomy than did team sport athletes. The team sport athletes scored significantly higher on agreeableness and sociotropy than did the individual sport athletes [3].

Along with this, Singer says that based on observations made on baseball athletes and tennis athletes, there were differences in personality aspects of team sport with individual sports athletes [4]. According to Williams \& Reilly (2000) based on the results of the study of the sport of football there are some psychological characteristics that need to be possessed by young athletes, namely control of the level of arousal, high confidence, focus on high tasks and the capacity to perform fully spirit [5].
Literature studies show an effect on success. A very important translation of his long achievements in sports and identification. Furthermore, the amount of personality is due to genetic and environmental integration [1]. With regard to research on the topic, the existence of a competitive athlete's personality characteristics has been controversy among researchers. Vealey (2002) has confirmed the absence of personality profiles for athletes, once there is no difference that can be distinguished between athletes and non-athletes [6].

Vanden Auweele, Nys, Rzewnicki, \& Van Mele, (2001) reported that are no different from non-athletes with regard to extroversion in three different apparatus (16 PF, EPI and EPQ), becoming a strong results for personality research [7].

Unlike the authors mentioned above, Karimi \& Besharat [2], Singer,et al [4]. Reported that the team sports presents some psychological characteristics that distinguish him from individual sports. Among these differences, the authors consider that individual sport athletes scored significantly higher on conscientiousness and autonomy than did team sport athletes. The team sport athletes scored significantly higher on agreeableness and sociotropy than did the individual sport athletes. No significant difference was found between the two groups on neuroticism, extraversion and openness. It can be concluded that athletes' personality characteristics are different in individual and team sports $[2,4]$.

Some studies have shown the positive effect of sport on personality, on the contrary some have shown no positive effect of sport on personality. Some believe that team athletes are more extraversion, with higher anxiety and dependency, on the contrary individual athletes are more introspection and with higher self-confidence. Then the study of athletes' personality characteristics, either individual or team sport athletes provide an opportunity for sport counselor, coaches and specialists to discover and select the talented ones for guiding them to the highest level of skills.

The main purpose of the present study is to survey and diagnose athletes' personality characteristics in individual and team sports and to compare their personality characteristics with each other. The component of personality including 20 indicators: achievement; adaptability; competitiveness; conscientiousness; visualization; intuition; goal setting; managing pressure; self-efficacy; fear of failure control; flow; stress management; emotions; self-talk; self-awareness; ethics; 
empathy; relationships; power; aggressiveness; and impression management.

\section{METHODOLOGY}

The method used in this research is survey method with ex post facto research design. This is used to determine the psychological condition of athletes PPLOP Central Java. A total of 197 athletes (105 individual, 92 team, 131 males, and 66 females) from individual sports (taekwondo, swimming, pencak silat, wushu, boxing, lifting, wrestling, karate, track and field, judo, archery, table tennis, climbing, and roller skaters) and team sports (football, canoeing, volley ball, basketball, and sepak takraw) composed the sample. All individuals were informed about the objectives of the research and that data would only be used for research purposes and generally analyzed, and they signed a consent form to participate in this study. Samples obtained from athletes who are at the Central Training Center for Student Sport (PPLOP) of Central Java province.

The instrument used was the reviewed Indonesian version of the Sport Personality Questionnaires (SPQ-20) (myskillprofile.com) containing 168 questions with response possibilities statement describes their behavior using a 5-point likert Scale (never/almost never, occasionally, fairly often, very often and always/almost always), and being applied just once, and also have 0,6 to 0,8 scale reliabilities [8]. The SPQ20 assesses 20 dimensions of mental toughness covering 4 key areas: confidence and resilience, achievement drive and competitiveness, power and aggressiveness, and interaction and sportsmanship.

Procedure Questionnaires were administered to subjects within 15-20 minutes and the nature of study was described at the top of the questionnaire to the subjects. Each question item took approximately 30 second to complete. Subjects who had completed the personal information section represented that they had given their consent to provide the data under the condition of anonymity. Investigator collected the questionnaires from each coach after completing the questionnaire. A total of 197 questionnaires were collected.

For the analysis of the personality characteristics comparison between individual and team sports, the descriptive analysis was initially used (average and standard deviation) for the behavior of each variable to be studied. In order to describe the data and differences the t-test was used. At the significance level of 0.05 was used in order to determine the differences between study groups. The statistical program used was the SPSS version 19.0.

\section{RESULT AND DISCUSSION}

\section{A. Socio-demographic characteristics}

The socio-demographic sample is shown in Table 1, ie: the total number of samples of 197 athletes (105 individual, 92 team, 131 males, and 66 females) from individual sports (taekwondo, swimming, pencak silat, wushu, boxing, lifting, wrestling, karate, track and field, judo, archery, table tennis, climbing, and roller skaters) and team sports (football, canoeing, volley ball, basketball, and sepak takraw)
TABLE I. : GENERAL CHARACTERISTICS OF THE SAMPLE $(\mathrm{N}=197)$

\begin{tabular}{|l|c|c|c|}
\hline & Individual & Team & Total \\
\hline $\mathrm{n}$ (Men) & 63 & 68 & 131 \\
\hline $\mathrm{n}$ (Female) & 42 & 24 & 66 \\
\hline $\mathrm{n}$ (Total) & 105 & 92 & 197 \\
\hline
\end{tabular}

\section{B. The differences between individual and team sport}

Can be seen in table 2 there are not many differences in each group based on the average score. The variables that most differed in the averages were: Conscientiousness (3,67 and 3,93 points); Flow (3,63 and 3,39 points); Self-awareness $(3,46$ and 3,68); Ethics (3,54 and 3,92) and Power (2,58 and 2,88). While, the variables that show smallest differences were: Goal setting (3,33 and 3,33 points); Stress management (3,50 and 3,46 points) and Relationship (3,16 and 3,21 points).

In order to verify these differences statistically, the Student's t-test was applied (table 2) and the sample of individual sport was significantly distinguished from team sports $(\mathrm{p}<0.05)$ in five out of 21 variables of the SPQ20 instrument: Conscientiousness, Flow, Self-awareness, Ethics and Power.

TABLE II. : AVERAGE AND STANDARD DEVIATION OF PERSONALITY VARIABLES OF INDIVIDUAL AND TEAM SPORTS

\begin{tabular}{|l|c|c|c|c|c|c|}
\hline \multirow{2}{*}{ Variable } & \multicolumn{2}{|c|}{ Individual } & \multicolumn{2}{c|}{ Team } & \multirow{2}{*}{$" t "$} & \multirow{2}{*}{ P } \\
\cline { 2 - 5 } & $\mathrm{X}$ & $\mathrm{SD}$ & $\mathrm{X}$ & $\mathrm{SD}$ & & \\
\hline Achievement & 3,31 & 0,56 & 3,46 & 0,77 & $-1,629$ & 0,105 \\
\hline Adaptability & 3,49 & 0,72 & 3,71 & 1,48 & $-1,406$ & 0,161 \\
\hline Competitiveness & 3,78 & 0,73 & 3,93 & 0,79 & $-1,454$ & 0,148 \\
\hline Conscientiousness & $\mathbf{3 , 6 7}$ & $\mathbf{0 , 7 7}$ & $\mathbf{3 , 9 3}$ & $\mathbf{0 , 8 6}$ & $\mathbf{- 2 , 2 6 4}$ & $\mathbf{0 , 0 2 5}$ \\
\hline Visualization & 3,63 & 0,62 & 3,72 & 0,82 & $-0,990$ & 0,324 \\
\hline Intuition & 3,03 & 0,58 & 3,15 & 0,82 & $-1,154$ & 0,250 \\
\hline Goal Setting & 3,33 & 0,51 & 3,33 & 0,84 & $-0,097$ & 0,923 \\
\hline Managing Pressure & 3,11 & 0,60 & 3,00 & 0,69 & 1,084 & 0,280 \\
\hline Self-Efficacy & 3,44 & 0,57 & 3,32 & 0,73 & 1,185 & 0,237 \\
\hline Fear of Failure & 3,13 & 0,74 & 3,23 & 0,90 & $-0,867$ & 0,387 \\
\hline Control & $\mathbf{3 , 6 3}$ & $\mathbf{0 , 5 7}$ & $\mathbf{3 , 3 9}$ & $\mathbf{0 , 8 4}$ & $\mathbf{2 , 2 8 2}$ & $\mathbf{0 , 0 2 4}$ \\
\hline Flow & 3,50 & 0,53 & 3,46 & 0,81 & 0,367 & 0,714 \\
\hline Stress Management & 3,23 & 0,60 & 3,42 & 1,19 & $-1,485$ & 0,139 \\
\hline Emotions & 3,61 & 0,66 & 3,76 & 0,93 & $-1,300$ & 0,195 \\
\hline Self-Talk & $\mathbf{3 , 4 6}$ & $\mathbf{0 , 6 2}$ & $\mathbf{3 , 6 8}$ & $\mathbf{0 , 8 7}$ & $\mathbf{- 2 , 1 3 1}$ & $\mathbf{0 , 0 3 4}$ \\
\hline Self-Awareness & $\mathbf{3 , 5 4}$ & $\mathbf{0 , 6 0}$ & $\mathbf{3 , 9 2}$ & $\mathbf{1 , 2 0}$ & $\mathbf{- 2 , 8 7 5}$ & $\mathbf{0 , 0 0 4}$ \\
\hline Ethics & 3,14 & 0,61 & 3,32 & 1,33 & $-1,291$ & 0,198 \\
\hline Empathy & 3,16 & 0,45 & 3,21 & 0,69 & $-0,685$ & 0,494 \\
\hline Relationships & $\mathbf{2 , 5 8}$ & $\mathbf{0 , 6 5}$ & $\mathbf{2 , 8 8}$ & $\mathbf{0 , 8 6}$ & $\mathbf{- 2 , 7 7 0}$ & $\mathbf{0 , 0 0 6}$ \\
\hline Power & 2,77 & 0,74 & 2,94 & 0,86 & $-1,483$ & 0,140 \\
\hline Aggressiveness & 3,24 & 0,71 & 3,41 & 0,91 & $-1,497$ & 0,136 \\
\hline Impression & & & & & \\
\hline Management & 3,43 & \\
\hline
\end{tabular}

Based on these differences, individual sports showed higher significant point $(\mathrm{p}<0,05)$ only on variable Flow and lower in 
variables Conscientiousness, Self-awareness, Ethics and Power. In other hand, team sports presented higher significant point in variables Conscientiousness, Self-awareness, Ethics and Power and lower only on variable Flow. These data characterize individual sports with team sports in the point of view of personal relationships.

The data obtained in this study are not agreement with findings of Widyaningsih et al. (2018); Zourmand and Changzhu (2017) who verified that individual sports has been higher on variable achievement, conscientiousness, visualization, self-efficacy, self-talk, self-awareness, and ethics. Thus, team sport higher on the variable goal setting, imagery and self-talk $[9,10]$.

Environmental factors have an influence that makes a person similar to others because of the various experiences that he experienced. Environmental factors consist of cultural factors, social class, family, peers, and situations. Among the environmental factors that have a significant influence on personality are individual experiences as a result of a particular culture. Each culture has its own rules and sanction patterns of learned behavior, rituals and beliefs. This means that each member of a culture will have certain common personality characteristics $[6,11,13]$.

Comparing with other studies conducted by Nia and Ali Besharat (2010); Eagleton, McKelvie, and De Man (2007), who investigated and compared individual and team sports, the data found in the present study corroborate the fact that individual sports more achieve and control emotion with other $[14,15]$. There are also differences with data obtained by Auweele et all (2001) and Morgan and Costill (1996) for variable Achievement, Competitiveness, Empathy, Relation, Strength and Aggressiveness that presented no significant variations, while for the mentioned authors, team sports athletes were characterized by being more extroverted and by the fact that athletes presented lower stress level, which contrasted with data found in the present study $[7,16,17]$.

It should be underlined the inconsistency of results and conclusions in the comparison of athlete personality in each sport, generated by various studies conducted so far, caused by various ahs. The reason for this is that there are differences in the research instruments used (EPQ, 16 PF, FPI, POMS, EPI, and now SPQ20) that measure different variables and personality components and there can be no significant comparison between instruments. The number of intervened variables (social, educational, cultural and economic) can affect the differences and inconsistencies in the results obtained.

Although the results of this study proved inconsistent, the limitations in this study need to be understood. One limiting factor is that the sample used is the PPLOP athlete of Central Java and limited and it is important to note that the number of athletes in the school is very limited. Thus, the differences found between the groups should be understood.

In this case, the samples of athletes selected for this study do not represent the overall population of athletes in Indonesia, as there are some age ranges, social classes, cultures, ethnicities, races, educational levels that make generalization of data difficult. Therefore, the results obtained should be considered an indication of the possible differences between athletes in each sporting population, but in order for the results of this study to show a constant result in the entire population, further research should be conducted with a wider object.

Other limitation of the present study is the lack of knowledge of researches on the personality of PPLOP Central Java, Indonesia athletes performed with the SPQ-20. This argument makes it difficult to discuss variables that are compared with the personality dimension in other instrument personality. This aspect can be regarded as a limiting factor for personality studies, after discovering characteristics that identify specific study groups becomes increasingly difficult.

\section{CONCLUSIONS}

The goal of this research is to compare the personality characteristics of individual and team sports, and also present results contrary to the findings of previous research. However, it is clearly concluded that athletes are significantly different in the variables studied. People can run out of the results found, there are athletes in various sports is always different because each has different characteristics and possibilities from the social, geographic and culture. This verification demonstrates comprehensive data consistency and indicates the possible generalization of differences between individuals of both groups; the facts are worth investigating further.

In order to characterize high-level athletes personality can be studied and understood better, and for the development of better knowledge in this field, several studies on the following topics are suggested:

- Longitudinal studies that allow an evaluation of athlete personality development from the first year to high degree are achieved;

- To compare athletes from different performance levels with different non-athlete samples for better differentiation formations where the extracts appear more clearly.

\section{ACKNOWLEDGMENT}

To all PPLOP Central Java athletes who voluntarily participated in the data collection and to the members of technical commissions of the following sportive modalities that enabled the performance of this study: chief of PPLOP Central Java and coaches.

\section{REFERENCES}

[1] M. S. Allen, I. Greenlees, and M. Jones, "Personality in sport: A comprehensive review," International Review of Sport and Exercise Psychology, vol. 6, no. 1. pp. 184-208, 2013.

[2] M. Karimi and M. A. Besharat, "Comparison of hardiness, sociotropy and autonomy in team and individual sports and investigating the impact of these personality characteristics on sport achievement," in Procedia - Social and Behavioral Sciences, 2010, vol. 5, pp. 855-858.

[3] M. E. Nia and M. Ali Besharat, "Comparison of athletes' personality characteristics in individual and team sports," in Procedia - Social and Behavioral Sciences, 2010, vol. 5, pp. 808-812.

[4] R. N. Singer, H. a Hausenblas, and C. Janelle, Handbook of Sport Psychology. 2000. 
[5] A. M. Williams and T. Reilly, "Talent identification and development in soccer," J. Sports Sci., vol. 18, no. 9, pp. 657-667, 2000.

[6] R. S. Vealey, "Personality and sport behavior.," Advances in sport psychology (2nd ed.). pp. 43-74, 2002.

[7] Y. Vanden Auweele, K. Nys, R. Rzewnicki, and V. Van Mele, "Personality and the athlete," Chem. Eng. J., vol. 140, no. 1-3, pp. 157-164, 2001.

[8] Myers Briggs Foundation, "The Myers \&amp; Briggs Foundation MBTI ${ }^{\circledR}$ Basics,” The Myers \& Briggs Foundation, 2016. [Online]. Available: http://www.myersbriggs.org/my-mbti-personalitytype/mbti-

basics/home.htm?bhcp=1\%0Ahttp://www.myersbriggs.org/my-mbtipersonality-type/mbti-basics/.

[9] W. W. Widyaningsih, O. Woro, K. Handayani, and T. Hidayah, "Journal of Physical Education and Sports The Relationship between Personality of Single and Double Athletes of Badminton Toward Achievement Level in PB . Djarum," vol. 7, no. 1, pp. 1-6, 2018.

[10] G. Zourmand and Q. Changzu, "The Effect of Physical and Mental Anxiety Reduction Techniques on Athletes," J. Enginering Appl. Sci. vol. 12, no. 6, pp. 1474-1478, 2017

[11] R. R. McCrae and P. T. Costa, Jr., "The five-factor theory of personality," in Handbook of Personality: Theory and Research, no. 2, 1999, pp. 159-181.
[12] D. C. Funder, "Personality," Annu. Rev. Psychol., vol. 52, no. 1, pp. 197-221, 2001.

[13] E. Aidman and G. Schofield, "Personality and individual differences in sport," Sport Psychol. Theory, Appl. issues (2nd ed.)., vol. 60, no. 2014, pp. 22-47, 2004

[14] J. R. Eagleton, S. J. McKelvie, and A. De Man, "Extra Version and Neuroticism in Team Sport Participants, Individual Sport Participants, and Nonparticipants," Percept. Mot. Skills, vol. 105, no. 1, pp. 265275, 2007.

[15] M. E. Nia and M. Ali Besharat, "Comparison of athletes' personality characteristics in individual and team sports," Procedia - Soc. Behav. Sci., vol. 5, no. 2, pp. 808-812, 2010.

[16] W. P. Morgan and D. L. Costill, "Selected psychological characteristics and health behaviors of aging marathon runners: A longitudinal study," Int. J. Sports Med., vol. 17, no. 4, pp. 305-312, 1996.

[17] D. Vinson, K. Beeching, M. Morgan, and G. Jones, "Collaborative evaluation of individual and team performance in training and match environments using the Coach Logic online platform," Int. Sport Coach. J., vol. 4, pp. 47-62, 2017. 